\title{
The Role of Stress Echocardiography in Valvular Heart Disease: A Current Appraisal
}

\author{
James L. Gentry III Dermot Phelan Milind Y. Desai Brian P. Griffin \\ Robert and Suzanne Tomsich Department of Cardiovascular Medicine, Heart and Vascular Institute, \\ Cleveland Clinic, Cleveland, OH, USA
}

\section{Keywords}

Stress echocardiography · Exercise - Dobutamine - Valvular heart disease $\cdot$ Sports eligibility $\cdot$ Guidelines

\begin{abstract}
Stress echocardiography is a widely available, safe, low-cost, versatile imaging modality which is becoming increasingly recognized as a valuable tool in the assessment of patients with native and prosthetic left-sided valvular heart disease. It provides a quantitative assessment to help guide clinical decision-making when discordance exists between symptoms and severity of valve disease. Exercise (treadmill or bicycle) remains the preferred stress modality, but pharmacological augmentation with dobutamine can be used if needed. Low-dose dobutamine stress echocardiography is specifically valuable in patients with low-flow, low-gradient aortic stenosis when attempting to differentiate true severe aortic stenosis from pseudo-severe aortic stenosis. Stress echocardiography not only identifies high-risk features that indicate need for earlier surgery, it also provides useful information for the peri- and postoperative period, including long-term outcome, risk stratification to guide monitoring frequency, and offers guidance for eligibility in competitive sports participation. As research continues to expand the
\end{abstract}

\section{KARGER}

(C) 2017 S. Karger AG, Basel

E-Mail karger@karger.com

www.karger.com/crd utility of stress echocardiography in the management of patients with valvular heart disease, future research should focus on the recognition of newer parameters identifying high-risk features including subsequent validation in a large population.

(c) 2017 S. Karger AG, Basel

\section{Introduction}

Stress echocardiography is a widely available, safe, low-cost, versatile imaging modality which is becoming increasingly recognized as a valuable tool in the assessment of patients with valvular heart disease [1-4]. It can be especially helpful in patients with slowly progressive valvular heart disease, which can result in gradual unintentional adaptations including reduction in functional capacity, and a sedentary lifestyle [5]. The 2014 American College of Cardiology (ACC)/American Heart Association (AHA) Valvular Heart Disease Guidelines and 2012 European Society of Cardiology (ESC) Guidelines on the Management of Valvular Heart Disease recognize the use of exercise testing in asymptomatic severe valvular heart disease (ACC/AHA class IIa recommendations) to objectively (a) confirm the absence of symptoms, (b) evaluate

James L. Gentry III, MD

Robert and Suzanne Tomsich Department of Cardiovascular Medicine Heart and Vascular Institute, Cleveland Clinic, J3-5 9500 Euclid Avenue, Cleveland, OH 44195 (USA)

E-Mail gentryj@ ccf.org 
the hemodynamic response to exercise, and (c) provide guidance on prognosis $[6,7]$. Additionally, the European Association of Cardiovascular Imaging and the American Society of Echocardiography have recently published a joint document highlighting the use of stress echocardiography in nonischemic heart disease with a large proportion dedicated to valvular heart disease [8].

\section{Types of Stress Test}

Stress echocardiography can be performed before and after exercise (treadmill/bicycle), or, in specific situations such as low-flow, low-gradient (LFLG) aortic stenosis (AS), pharmacological stress using low-dose dobutamine (up to $20 \mu \mathrm{g} / \mathrm{kg} / \mathrm{min}$ ) to augment the cardiac output and determine the effect of this on the aortic valve (AV) area (AVA) and transvalvular pressure gradients (TPGs) [9]. In any stress test for the assessment of valve disease, comprehensive hemodynamic data and images are acquired at rest and subsequently at peak exercise. This enables to determine the hemodynamic impact of the valve lesion on the affected valve gradients and regurgitation, pulmonary pressures derived from tricuspid regurgitation velocity recordings, left ventricle (LV) and right ventricle (RV) size and function while also providing information on functional capacity. These parameters not only prognosticate but also provide insight into the significance of the valvular lesion under differing physiological loading conditions, can help determine the need for and timing of intervention, as well as provide objective evidence of functional limitations [9].

\section{Parameters Monitored during Stress}

Patients typically first undergo a full resting echocardiogram which includes standard assessment of biventricular volumes and function along with specific measurements of valve hemodynamics including TPGs, valve area, stroke volume, and resting RV systolic pressure (RVSP). Most of these measurements are repeated at peak stress with the addition of pertinent disease parameters as needed. Cycle ergometers have the advantage of allowing acquisition of measurements during stress, unlike treadmill testing where the measurements are made at the completion of exercise. It is important that whoever is performing the exercise study is aware of the hierarchy of priority of the individual parameters. For instance, in mitral stenosis (MS), the pressure gradients at peak exercise are often the initial parameters acquired at peak exercise whereas for mitral regurgitation (MR), this may be the LV size and function and severity of regurgitation at peak exercise. What constitutes an abnormal response to exercise will vary by valve lesion, age, and gender. Typically, a stress echocardiogram is considered abnormal if any of the following conditions occur: symptoms occur at lower than expected workload, exercise capacity is $<85 \%$ of that of an age- and gender-matched cohort, blood pressure (BP) falls in response to stress or is blunted (systolic BP rise $<20 \mathrm{~mm} \mathrm{Hg}$ ), development of exercise-induced pulmonary hypertension (PHTN) (RVSP $>60 \mathrm{~mm} \mathrm{Hg}$ ), increase in severity of valvular regurgitation, depression in LV ejection fraction (LVEF) including development of regional wall motion abnormalities, or development of significant sustained ventricular arrhythmias $[9,10]$. Additional specific abnormalities will be addressed in their respective sections.

\section{Aortic Valve}

\section{Aortic Stenosis}

Severe Aortic Stenosis

Current AHA/ACC and ESC guidelines recommend AV replacement (AVR) for severe AS with the onset of typical symptoms (class I), if asymptomatic when LVEF $<50 \%$ (class I), and reasonable regardless of symptoms in very severe AS (peak velocity $>5.0 \mathrm{~m} / \mathrm{s}$; mean pressure gradient $>60 \mathrm{~mm} \mathrm{Hg}$ ) (class IIa). The ACC/AHA and ESC guidelines also recommend AVR for asymptomatic severe AS patients who undergo exercise testing and develop symptoms (class I) or have an abnormal BP response (class IIa) $[6,7]$. The ESC guidelines also state that AVR can be considered in patients with low surgical risk if their mean aortic pressure gradient increases $>20 \mathrm{~mm}$ $\mathrm{Hg}$ with exercise compared to rest [7]. Lastly, AVR is reasonable for patients with moderate AS who are undergoing cardiac surgery for another indication (class IIa) $[6,7]$.

AS is a slowly progressive disease with average reduction in AVA of $0.1 \mathrm{~cm}^{2}$ per year, peak transvalvular velocity increase of $0.1-0.3 \mathrm{~m} / \mathrm{s} /$ year, and mean gradient increase of 3-10 mm Hg per year [11]. Fortunately, disease progression in most patients is slow; however, this results in patients unintentionally limiting their activities and thus remaining apparently "asymptomatic." However, recognition of symptoms is vitally important as it is associated with a $50 \%$ mortality at 2 years without surgical intervention $[12,13]$. At this time, no medical therapies are proven to prevent or slow the progression of AV stenosis, and definitive therapy involves AVR via surgical or a catheter-based approach for patients with elevated surgical mortality. According to data from the European As- 


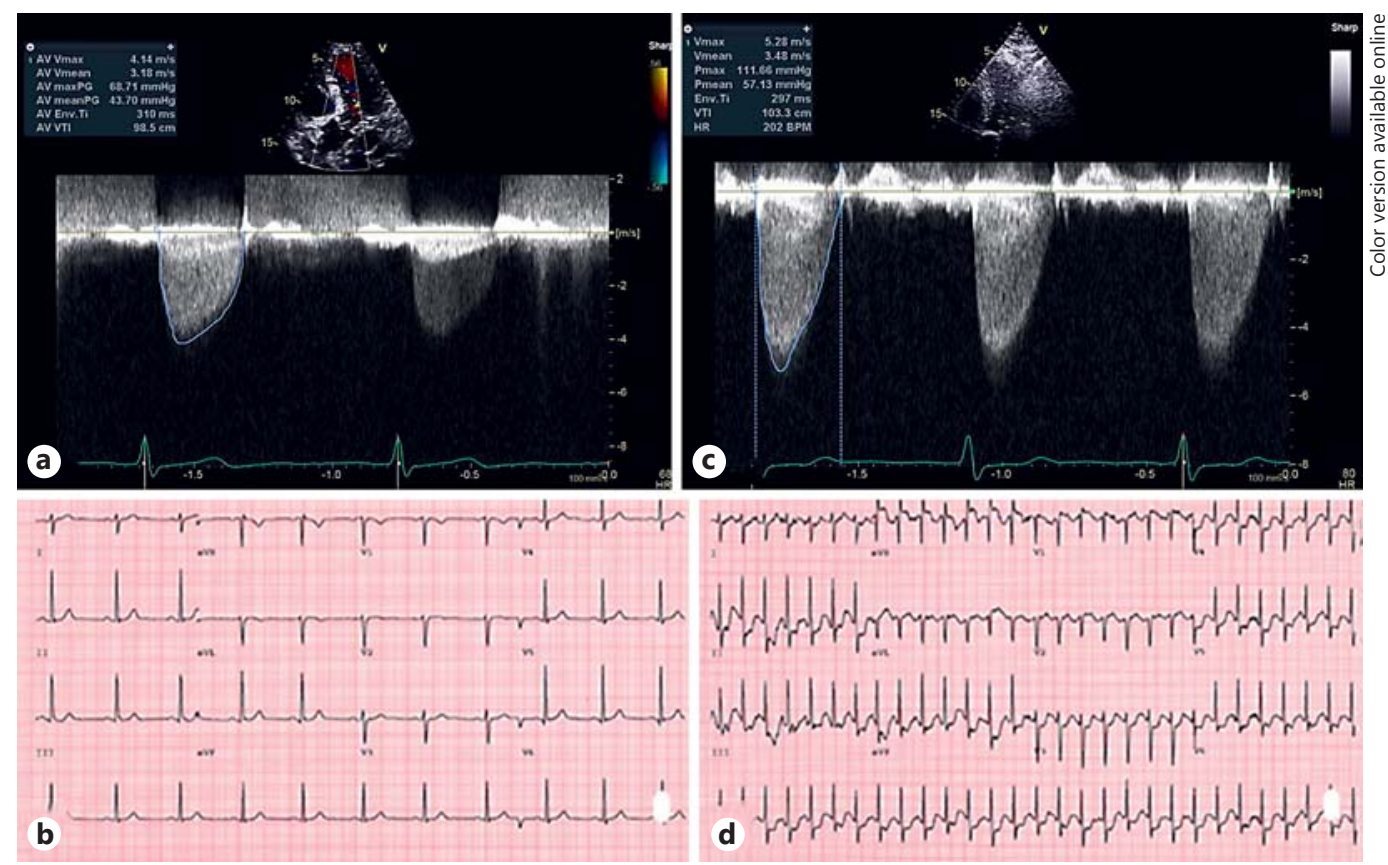

Fig. 1. Abnormal exercise stress echocardiogram: severe aortic stenosis in a 79-year-old male with asymptomatic severe aortic valve (AV) stenosis with an $\mathrm{AV}$ area of $0.83 \mathrm{~cm}^{2}$ by the continuity equation using velocity time integral (VTI). a Resting peak and mean AV gradients of 69 and $44 \mathrm{~mm} \mathrm{Hg}$ with a peak velocity of $4.1 \mathrm{~m} / \mathrm{s}$. b Resting electrocardiogram (ECG) with a blood pressure (BP) of
160/98 mm Hg. c Exercise peak and mean AV gradients of 112 and $57 \mathrm{~mm} \mathrm{Hg}$ with a peak velocity of $5.3 \mathrm{~m} / \mathrm{s}$. d Exercise ECG with diffuse $>2 \mathrm{~mm}$ ST segment depression and reciprocal elevation in aVR and fall in BP to $130 / 70 \mathrm{~mm} \mathrm{Hg}$ at peak exercise (8.6 metabolic equivalents). HR, heart rate. sociation for Cardiothoracic Surgery and the Society of Thoracic Surgeons in 2010, 30-day mortality for isolated AVR is approximately $3 \%$; however, rates vary based on experience, and the most recent in-hospital mortality data from our own institution in 2014 is significantly lower at $0.5 \%[14]$.

Use of stress echocardiography provides guidance in identifying "asymptomatic" patients who have gradually decreased their functional capacity or developed latent LV dysfunction that would benefit from AVR as compared to watchful waiting $[6,7]$. Symptom-limited exercise testing and low-dose dobutamine stress echocardiography (DSE) have both been proven to be safe to perform in patients with asymptomatic severe AS with appropriate physician supervision and close monitoring of the electrocardiogram and BP $[1,3,4]$. However, stress echocardiography is contraindicated and has no role in patients with symptomatic severe AS (class III) as there is a risk of cardiovascular catastrophe with stress in this population [6].

Based on prior studies of patients with asymptomatic severe AS who have undergone exercise stress echocar- diography (ESE), two thirds of tests are abnormal related to exercise parameters alone, including one third being attributable to the development of symptoms alone. An abnormal ESE is associated with an 8-fold higher risk of major adverse cardiac events, including a 5.5 times higher risk of sudden death, and increased longer-term mortality risk (up to 12 years) (Fig. 1) [15, 16]. Furthermore, a normal test provides reassurance with an excellent 1 -year prognosis without need for surgical intervention [16].

Stress echocardiography can also help identify highrisk patients that may require more frequent monitoring for disease progression. In 2 separate studies of patients with at least moderate AS (AVA $<1.2 \mathrm{~cm}^{2}$ ), the presence of an increase in mean AV gradient $>18-20 \mathrm{~mm} \mathrm{Hg}$ on exercise was associated with a 3.8-10 times higher risk of cardiovascular events (symptoms, hospitalization for heart failure, death, and/or need for AVR) [17]. The presence of exercise-induced PHTN (RVSP $>60 \mathrm{~mm} \mathrm{Hg}$ ) in patients with asymptomatic severe AS is also associated with a 2 times lower cardiac event-free survival compared to those without exercise-induced PHTN. Lastly, the ab- 
Table 1. Aortic stenosis (AS): abnormal stress echocardiography responses and contraindications

\begin{tabular}{|c|c|c|}
\hline & High-risk findings with stress & Contraindications \\
\hline Severe AS & $\begin{array}{l}\text { Asymptomatic } \\
\text { Development of symptoms }{ }^{\mathrm{a}} \\
\text { Exercise capacity }<85 \% \text { of predicted for age/gender }^{\mathrm{a}} \\
\text { Abnormal blood pressure response } \mathrm{e}^{\mathrm{a}} \\
\text { Decline in left ventricular ejection fraction } \\
\text { Increase in mean gradient }>18-20 \mathrm{~mm} \mathrm{Hg} \\
\text { Increase in right ventricular systolic pressure }>60 \mathrm{~mm} \mathrm{Hg} \\
\text { Absence of contractile reserve } \\
\text { Slow heart rate recovery }\end{array}$ & $\begin{array}{l}\text { Severe symptomatic AS } \\
\text { Very severe AS }\end{array}$ \\
\hline $\begin{array}{l}\text { Low-flow, } \\
\text { low-gradient AS }\end{array}$ & Absence of contractile reserve & \\
\hline Moderate AS & $\begin{array}{l}\text { If resting mean gradient }>35 \mathrm{~mm} \mathrm{Hg} \text {, exercise-induced } \\
\text { increase in mean gradient }>20 \mathrm{~mm} \mathrm{Hg}\end{array}$ & \\
\hline
\end{tabular}

sence of contractile reserve is indicative of intrinsic myocardial dysfunction and portends both a worse event-free survival and a higher risk of early postoperative LV decompensation [18]. The absence of LV contractile reserve is defined as the absence of an exercise-induced increase in LVEF, stroke volume increase of $<20 \%$ or, more sensitively, as a $<2 \%$ increase in global longitudinal strain (GLS) $[18,19]$. Most of these high-risk features (Table 1) are not currently recognized as an indication for AVR, but if present at least indicate a patient population that should be followed more closely and may require AVR sooner than those without these features.

Low-Flow, Low-Gradient Aortic Stenosis

Patients with reduced systolic function, AVA $<1.0$ $\mathrm{cm}^{2}$, and mean AV gradient $<40 \mathrm{~mm} \mathrm{Hg}$ are classified as LFLG-AS. This group is comprised of individuals with both true severe AS who would benefit from AVR and pseudo-severe AS who benefit from medical therapy but not AVR. Patients with pseudo-severe AS make up approximately $30 \%$ of patients classified as LFLG-AS. These patients have an inappropriately low AVA $<1.0 \mathrm{~cm}^{2}$ occurring in the setting of impaired LV function with resultant submaximal opening of a mildly-to-moderately stenotic valve $[20,21]$. Low-dose DSE plays an important role in differentiating pseudo- from true severe AS (Fig. 2) [22]. If contractile reserve is present with stress testing ( $>20 \%$ increase in stroke volume), patients with true severe AS may increase their mean AV gradient to $>40 \mathrm{~mm}$
$\mathrm{Hg}$ while maintaining an AVA $<1.0 \mathrm{~cm}^{2}$. In contrast, the AVA will increase to $>1.0-1.2 \mathrm{~cm}^{2}$ with only a minimal increase in AV gradients in patients with pseudo-severe AS [23]. The presence of contractile reserve has additional prognostic value for patients undergoing AVR being associated with a $5 \%$ perioperative morality if present compared to $32 \%$ if absent [22].

However, the lack of contractile reserve can make it challenging to differentiate true severe AS from pseudosevere AS. Emerging research proposes the use of a projected AVA $\left(\mathrm{AVA}_{\text {proj }}\right)$ to accurately discriminate between these two entities for patients with both reduced and preserved systolic function $[10,20,21,24,25]$. AVA $A_{\text {proj }}$ calculates what the AVA should be at a normal transvalvular flow rate (Q) of $250 \mathrm{~mL} / \mathrm{s}$ and allows comparison among different patients. Flow rates at different stages of the lowdose DSE protocols are used to derive the compliance of the valve/AVA from regression of the created line (Fig. 3). An AVA $A_{\text {proj }}$ value $>1.2 \mathrm{~cm}^{2}$ is consistent with pseudo-severe AS while $<1.0 \mathrm{~cm}^{2}$ is defined as true severe AS. This tool has been validated to not only define severity of stenosis but also to predict adverse events (higher risk with lower $\mathrm{AVA}_{\text {proj; }}$ HR 1.32 per $0.1-\mathrm{cm}^{2}$ decrease in $\mathrm{AVA}_{\text {proj }}$ ) in both preserved and depressed ejection fraction through work performed in the TOPAS (True or Pseudo Severe Aortic Stenosis) trial [20, 21].

Paradoxical LFLG-AS has also recently been recognized as a separate group of patients with impaired outcome and is defined as having a mean transaortic gradient
140

Cardiology 2017;137:137-150 DOI: $10.1159 / 000460274$
Gentry III/Phelan/Desai/Griffin 

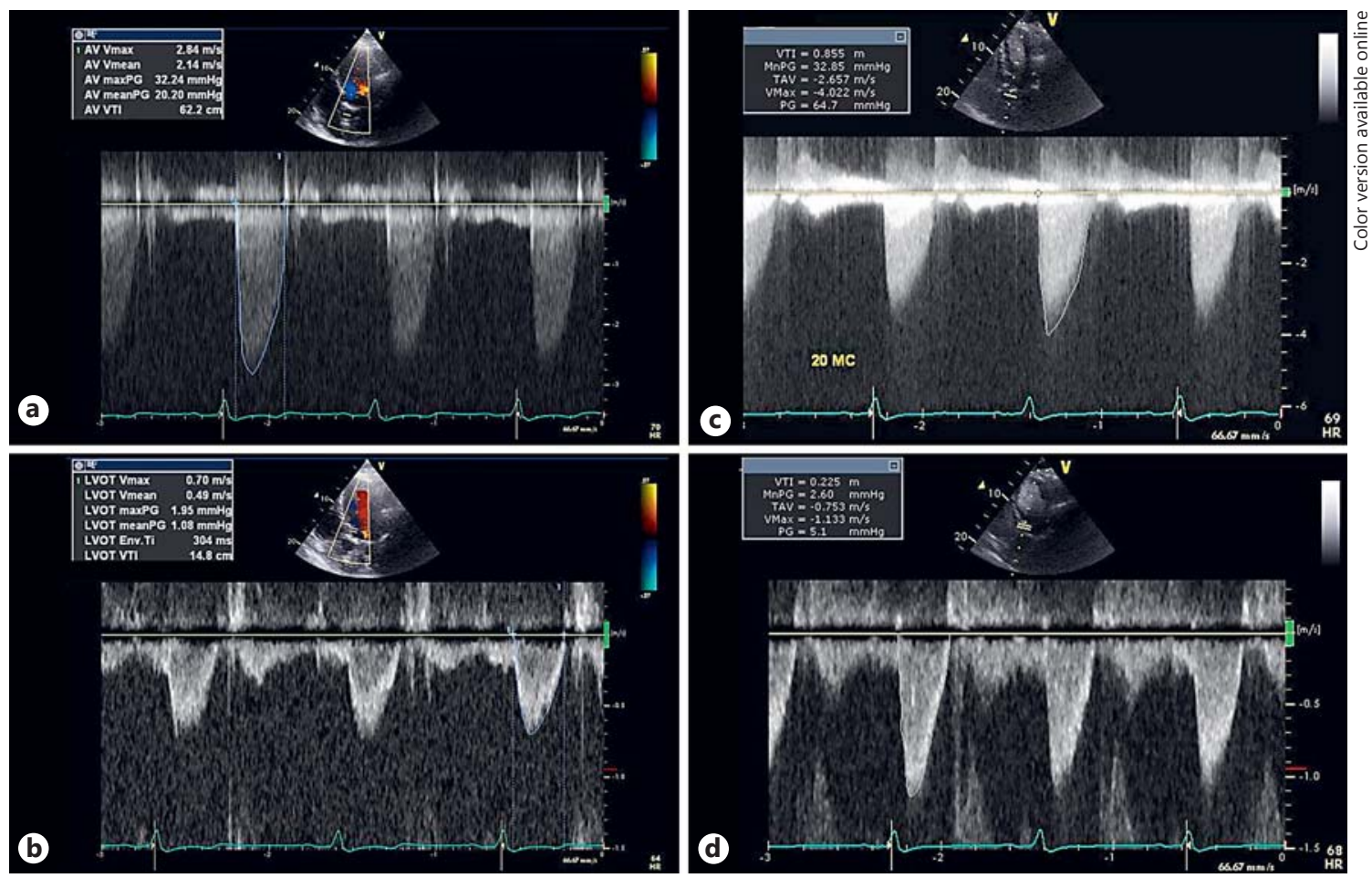

Fig. 2. Low-dose dobutamine stress echocardiogram: low-flow, low-gradient severe aortic stenosis in a 69-year-old male with systolic dysfunction (left ventricular ejection fraction 36\%) with a low stroke volume index $\left(25 \mathrm{~mL} / \mathrm{m}^{2}\right)$ and an aortic valve $(\mathrm{AV})$ area of $0.91 \mathrm{~cm}^{2}$ by continuity by velocity time integral (VTI) (left ventricular outflow tract [LVOT] diameter $2.2 \mathrm{~cm}$ ). a Resting peak and mean AV gradients of 32 and $20 \mathrm{~mm} \mathrm{Hg}$, respectively, with a peak velocity of $2.84 \mathrm{~m} / \mathrm{s}$. b Resting LVOT-VTI of $14.8 \mathrm{~cm}$ consistent with a low output state. c Dobutamine at $20 \mu \mathrm{g} / \mathrm{kg} / \mathrm{min}$ with peak and mean AV gradients of 65 and $33 \mathrm{~mm} \mathrm{Hg}$, respectively, and a peak velocity of $4.0 \mathrm{~m} / \mathrm{s}$. d Increase in LVOT-VTI to $22.5 \mathrm{~cm}$ consistent with the presence of contractile reserve and a calculated AV area of $1.0 \mathrm{~cm}^{2}$ by continuity by VTI.

$$
\mathrm{AVA}_{\text {proj }}=\frac{\mathrm{AVA}_{\text {peak }}-\mathrm{AVA}_{\text {rest }}}{\mathrm{Q}_{\text {peak }}-\mathrm{Q}_{\text {rest }}} \times\left(250-\mathrm{Q}_{\text {rest }}\right)+\mathrm{AVA_{ \text {rest } }}
$$

Fig. 3. Projected aortic valve area $\left(\mathrm{AVA}_{\text {proj }}\right)$ equation. $\mathrm{Q}$, transvalvular flow rate.

$<40 \mathrm{~mm} \mathrm{Hg}$ and AVA $<1.0 \mathrm{~cm}^{2}$ with a preserved ejection fraction $(>50 \%)$, but with a reduced stroke volume index $\left(<35 \mathrm{~mL} / \mathrm{m}^{2}\right)$. These patients are typically elderly with a history of hypertension which over time causes marked LV hypertrophy, small LV size, and an ensuing diminished stroke volume index [26]. Traditionally, DSE has not been used in this patient population with the historical exception of being used in the original transcatheter AVR trials to achieve a mean gradient of $40 \mathrm{~mm} \mathrm{Hg}$ or peak velocity of $4.0 \mathrm{~m} / \mathrm{s}$ required for trial inclusion [27,
28]. However, recent data by Clavel et al. [29] have shown that $\mathrm{AVA}_{\text {proj }}$ performed superior to alternative stenotic indices in differentiating true severe AS from pseudo-severe AS in patients with paradoxical LFLG-AS. However, this is not currently recommended for use in clinical practice as these findings have not been validated in any large population study, and there is a paucity of safety data for use of low-dose DSE in these patients.

\section{Moderate Aortic Stenosis}

ESE also has potential utility in patients with moderate AS to help determine appropriate clinical follow-up [30]. Currently, the ACC/AHA recommends repeating an echocardiogram in 1-2 years for moderately-severe AS (stage B) [6]. However, higher-risk patients have been identified with resting mean AV gradients $>35 \mathrm{~mm} \mathrm{Hg}$ and exercise-induced gradient augmentation in mean AV gradient $>20 \mathrm{~mm} \mathrm{Hg}$ who are at significantly higher risk for requiring surgical AVR or death during a mean fol- 
low-up of 20 months. Based on this research, Marechaux et al. [30] proposed that patients with a resting mean gradient $>35 \mathrm{~mm} \mathrm{Hg}$ with an exercise-induced gradient augmentation $<20 \mathrm{~mm} \mathrm{Hg}$ should be followed by at least yearly echocardiograms and every 6 months if their exercise-induced mean gradient increases $>20 \mathrm{~mm} \mathrm{Hg}$. It should also be noted that it is vitally important to verify that AV gradients are accurately obtained from multiple acoustic windows, including apical 5-chamber, suprasternal, and right parasternal views to ensure the highest $\mathrm{AV}$ velocities are being obtained both at rest and with stress. Failure to obtain the highest velocities results in underestimation of the severity of the valve disease. Other limitations also include patients being imaged by different sonographers and/or different ultrasound machines.

\section{Aortic Regurgitation}

Chronic Severe Aortic Regurgitation

The ACC/AHA and ESC guidelines recommend surgical AVR for patients with symptomatic severe aortic regurgitation (AR) (class I), asymptomatic chronic severe AR with LV systolic dysfunction ( $\mathrm{LVEF}<50 \%$ ) where the dysfunction is thought to be related to AR (class I), or those with moderate (ACC/AHA only) or severe AR undergoing cardiac surgery for other indications (class IIa and I, respectively). AVR is also reasonable in severe AR with normal LV systolic function but severe LV dilation (LV end-systolic diameter [LVESD] $>50 \mathrm{~mm}$ or indexed LVESD $>25 \mathrm{~mm} / \mathrm{m}^{2}$ or LV end-diastolic diameter [LVEDD] $>70 \mathrm{~mm}$ [ESC only]) (class IIa) and can be considered in severe AR with normal LVEF but with progressive severe LV dilation (LVEDD $>65 \mathrm{~mm}$; ACC/AHA only) (class IIb) [6, 7].

Similar to patients with AS, exercise testing can unmask symptomatic patients previously classified as being asymptomatic or with equivocal symptoms who would receive a significant mortality benefit from AVR compared to delaying surgery. However, the progression of disease in asymptomatic severe chronic AR is much slower based on the natural history of the disease with an estimated annual progression (requiring AVR or death) rate of approximately 6\% [31]. Monitoring for disease progression is particularly important in patients with a dilated LV in order to perform timely intervention prior to the development of irreversible dysfunction. In patients who do not meet criteria for AVR, ESE can also be used to assess contractile reserve to detect subclinical LV dysfunction. Despite being asymptomatic or only mildly symptomatic, the absence of contractile reserve is more predictive of the development of systolic dysfunction both at follow-up (medical therapy) and postoperatively than parameters obtained at rest $[32,33]$. Patients with severe AR may demonstrate an exercise-induced fall in LVEF due to the hemodynamic consequences of volume overload and increased afterload. This finding is nonspecific, and the reliability of this finding in predicting outcome is controversial. This topic was not specifically addressed in the most recent ACC/AHA and ESC guidelines, but, based on previous valve guidelines, it was considered a class III indication for surgical AVR when found in isolation [34].

$\mathrm{RV}$ function has also been increasingly recognized as an important prognostic parameter. Exercise tricuspid annular plane systolic excursion (TAPSE), a simple parameter used to assess RV function, which can easily be performed with exercise, was found to be independently associated with the need for earlier AVR in patients with asymptomatic severe AR if $<21 \mathrm{~mm}$ [35]. Other recent research has proposed parameters such as exercise tissue Doppler peak systolic mitral annular velocity and atrioventricular plane displacement, as well as exercise global longitudinal function, which will require further evaluation prior to acceptance in clinical practice $[36,37]$. While none of these exercise parameters have been included in guidelines for routine clinical use, these parameters could be considered for use to help anticipate surgical timing in higher-risk patients with parameters nearing recommendations for AVR, including LVEF 50-55\% or LVESD approaching $50 \mathrm{~mm}$ or indexed LVESD 25 $\mathrm{mm} / \mathrm{m}^{2}$ (Table 2) [38].

\section{Mitral Valve}

\section{Mitral Stenosis}

Percutaneous mitral balloon commissurotomy (PMBC) or mitral valve (MV) surgery is recommended for patients with severe symptomatic MS (ACC/AHA and ESC; class I) [6, 7]. Per the ESC guidelines, PMBC can also be considered in asymptomatic patients with $\mathrm{MV}$ area (MVA) $<1.5 \mathrm{~cm}^{2}$ only if they have favorable characteristics and either a high thromboembolic risk (history of embolism, dense spontaneous contrast in the left atrium, or recent or paroxysmal atrial fibrillation) or a high risk of hemodynamic decompensation (rest RVSP $>50$ $\mathrm{mm} \mathrm{Hg}$, need for major noncardiac surgery, or desire for pregnancy) [7]. Additionally, the ACC/AHA recognizes the consideration of PMBC for symptomatic patients with MVA $>1.5 \mathrm{~cm}^{2}$ if there is evidence of hemodynami- 
Table 2. Chronic aortic regurgitation (AR): abnormal stress echocardiography and contraindications

\begin{tabular}{lll}
\hline & High-risk findings with stress & Contraindications \\
\hline Severe AR & $\begin{array}{l}\text { Asymptomatic } \\
\text { Development of symptoms }\end{array}$ & Severe symptomatic AR \\
& Exercise capacity $<85 \%$ of predicted for age/gender \\
& LVEF $50-55 \%$, LVED approaching $50 \mathrm{~mm}$, or indexed LVESD \\
& of $25 \mathrm{~mm} / \mathrm{m}^{2}$ and absence of contractile reserve \\
& Exercise tricuspid annular plane systolic excursion $<21 \mathrm{~mm}$ \\
& Absence of contractile reserve & \\
\hline
\end{tabular}

LVEF, left ventricular ejection fraction; LVESD, left ventricular end-systolic diameter.

${ }^{\text {a }}$ Indication for aortic valve surgery.

cally significant MS during exercise (pulmonary capillary wedge pressure $>25 \mathrm{~mm} \mathrm{Hg}$ or mean valve gradient increase $>15 \mathrm{~mm} \mathrm{Hg}$ ) (class IIb). Per ACC/AHA guidelines, MV surgery is only indicated in patients who are severely symptomatic (New York Heart Association [NYHA] class III/VI), without high surgical risk, and are not a candidate for PMBC (class I) [6]. The ESC has similar recommendations with the exception of not quantifying the severity of symptoms required for surgery [7]. In asymptomatic severe MS, the ACC/AHA recommends undergoing MV surgery if undergoing cardiac surgery for other indications (class I; class IIb for moderate MS [MVA $1.6-2.0 \mathrm{~cm}^{2}$ ]) and PMBC in very severe MS in the presence of favorable anatomy (class IIa) [6].

Stress echocardiography is most valuable in MS when discordance exists between symptoms and the severity of stenosis (symptomatic moderate MS or asymptomatic severe MS) to assess for a clinically relevant increase in mean MV gradient and/or pulmonary artery pressures with stress (ACC/AHA class I) [6, 7]. MV gradients and pulmonary artery pressures are influenced by exercise through increases in cardiac output and heart rate, and a decrease in diastolic filling time leading to an exponential rise in left atrial and pulmonary capillary pressures [39]. This highlights why echocardiography performed at rest may not reflect the precise severity of the valvular disease. Since ESE is more physiologic, it is the favored modality and reveals symptoms in as high as $46 \%$ of patients with moderate-to-severe MS previously considered asymptomatic [40]. However, DSE may also be used if exercise cannot be performed.

Elevation in pulmonary vascular resistance can occur as an adaptive response to protect the lungs from the development of pulmonary edema and can be used as an indicator of the hemodynamic consequences of MS. With

Echocardiograpy in Valvular Heart Disease exercise, this adaptive response is unable to remain compensated and becomes a high-risk feature when RVSP becomes elevated $>60 \mathrm{~mm} \mathrm{Hg}[6,34]$. However, Brochet et al. [40] recently revealed that despite similar peak RVSP levels, the presence of a rapid rise in RVSP was superior (2-fold) at predicting the development of dyspnea and/or referral for valvular intervention than the presence of the rise in RVSP $>60 \mathrm{~mm} \mathrm{Hg}$ alone. Specifically, the higherrisk groups developed an early rise of $>90 \%$ increase in RVSP at $60 \mathrm{~W}$ of exercise. If DSE is performed, a mean gradient $>18 \mathrm{~mm} \mathrm{Hg}$ identifies a subgroup of individuals with a significantly higher proportion of clinical events, including hospitalization for dyspnea or arrhythmias, requiring MV intervention, or death. Conversely, if the mean gradient is $<18 \mathrm{~mm} \mathrm{Hg}$, this predicts an uneventful clinical course justifying a more conservative approach [41]. Exercise may also occasionally identify patients who are symptomatic at exercise despite less severe degrees of MS in whom MR increases significantly with exercise and may be the more important lesion leading to symptoms [42].

Current guidelines define hemodynamically significant MS with exercise as an increase in mean MV gradient $>15 \mathrm{~mm} \mathrm{Hg}$ or pulmonary capillary wedge pressure $>25$ $\mathrm{mm} \mathrm{Hg}$; however, there are limited data available to support these specific parameters. Furthermore, the most recent ACC/AHA guidelines removed part of former class IIb recommendations in which exercise-induced PHTN (RVSP $>60 \mathrm{~mm} \mathrm{Hg}$ ) was used as a parameter in the evaluation for PMBC [6].

Presently, the ACC/AHA and ESC guidelines recommend serial transthoracic echocardiograms for asymptomatic severe MS every 1-2 years unless MVA is $<1.0$ $\mathrm{cm}^{2}$, in which the ACC/AHA recommends a repeat echocardiogram in $<1$ year $[6,7]$. However, the valvular guide-

Cardiology 2017;137:137-150 DOI: $10.1159 / 000460274$ 
Table 3. Mitral stenosis (MS): abnormal stress echocardiography responses and contraindications

\begin{tabular}{lll}
\hline & High-risk findings with stress & Contraindications \\
\hline Severe MS & Asymptomatic & Severe symptomatic MS \\
& Development of symptoms ${ }^{\text {a }}$ & \\
& Exercise capacity $<85 \%$ of predicted for age/gender ${ }^{\mathrm{a}}$ & \\
& Increase in mean gradient to $>18 \mathrm{~mm}$ HG (DSE) or $>15 \mathrm{~mm} \mathrm{Hg}$ (ESE) \\
& RVSP $>60$ mm Hg at peak exercise & \\
& $>90 \%$ increase in RVSP at 60 -W exercise &
\end{tabular}

Moderate MS Increase in mean gradient $>15 \mathrm{~mm} \mathrm{Hg}^{\mathrm{b}}$

DSE, dobutamine stress echocardiography; ESE, exercise stress echocardiography; RVSP, right ventricular systolic pressure.

${ }^{a}$ Indication for percutaneous mitral balloon commissurotomy (PMBC) if symptomatic and favorable anatomy. Mitral valve surgery is only indicated if patients are symptomatic (NYHA class III/IV only per ACC/AHA guidelines), not at high risk for surgery, and are either not candidates for PMBC or failed prior PMBC. ${ }^{\mathrm{b}}$ Indication for consideration of PMBC only if also symptomatic.

lines do not incorporate use of all of the previously described high-risk features (Table 3; Fig. 4). Individuals with these high-risk features are indicative of impaired exercise tolerance and should receive a more aggressive approach including more frequent monitoring at a minimum.

\section{Mitral Regurgitation}

Chronic Primary Mitral Regurgitation

The ACC/AHA and ESC valve guidelines recommend MV surgery as a class I indication if the patient is (a) symptomatic with LVEF $>30 \%$ or (b) asymptomatic with LV dysfunction (LVEF $30-60 \%$ or LVESD $>40 \mathrm{~mm}$ ). MV repair for asymptomatic patients with preserved LV function is also felt to be reasonable (class IIa) by the ACC/ AHA and ESC if there is (a) high likelihood of successful repair (>95\%) and low mortality rate $(<1 \%)$, (b) a new onset of atrial fibrillation, or (c) presence of PHTN at rest (RVSP $>50 \mathrm{~mm} \mathrm{Hg}$ ) $[6,7]$. In this same patient population, MV repair can be considered (class IIb; ESC) if there is (a) presence of left atrial dilatation (volume index $>60$ $\mathrm{mL} / \mathrm{m}^{2}$ body surface area) or (b) presence of exercise-induced RVSP $>60 \mathrm{~mm} \mathrm{Hg}$ [7]. In the 2014 ACC/AHA valve guidelines, the presence of exercise-induced RVSP $>60 \mathrm{~mm} \mathrm{Hg}$ was specifically excluded after previously being part of a class IIa indication in 2006 [6,34]. In patients with LVEF $<30 \%$, MV surgery can also be considered (class IIb) for symptomatic patients based on the ACC/ AHA guidelines and only if refractory to medical therapy according to the ESC guidelines [6, 7]. Only the ACC/ AHA guidelines address the topic of patients with MR undergoing cardiac surgery for another indication and recommend concomitant MV surgery for severe MR (class I) and moderate MR (class IIa) [6].

Chronic Functional Mitral Regurgitation

Functional or secondary MR refers to a structurally normal MV with dysfunction being secondary to leaflet tethering, abnormal papillary muscles, LV dyssynchrony, and/or reduced contractility/ischemia. Ischemic MR typically occurs when the posterior mitral leaflet is tethered impairing MV coaptation and results in an eccentric, posteriorly directed MR jet lending itself to underestimation of regurgitant severity. MV surgery only reestablishes MV competence without addressing the primary disease process, thus outcomes in these circumstances are associated with a higher operative mortality and worse long-term prognosis compared to an equivalent surgery for primary MR [7]. For this reason, MV surgery recommendations are less aggressive compared to chronic primary MR and typically pertain to patients with persistent symptoms despite optimal medical therapy or patients undergoing cardiac surgery for another indication.

Per ACC/AHA guidelines, it is reasonable (class IIa) to undergo MV surgery for secondary severe MR at the time of either coronary artery bypass grafting or AVR and can be considered (class IIb) for moderate secondary MR if undergoing another cardiac surgery [6]. The ESC guidelines offer stronger recommendations with a recommendation to undergo MV surgery (class I) if they are undergoing coronary artery bypass grafting and LVEF is $>30 \%$, 

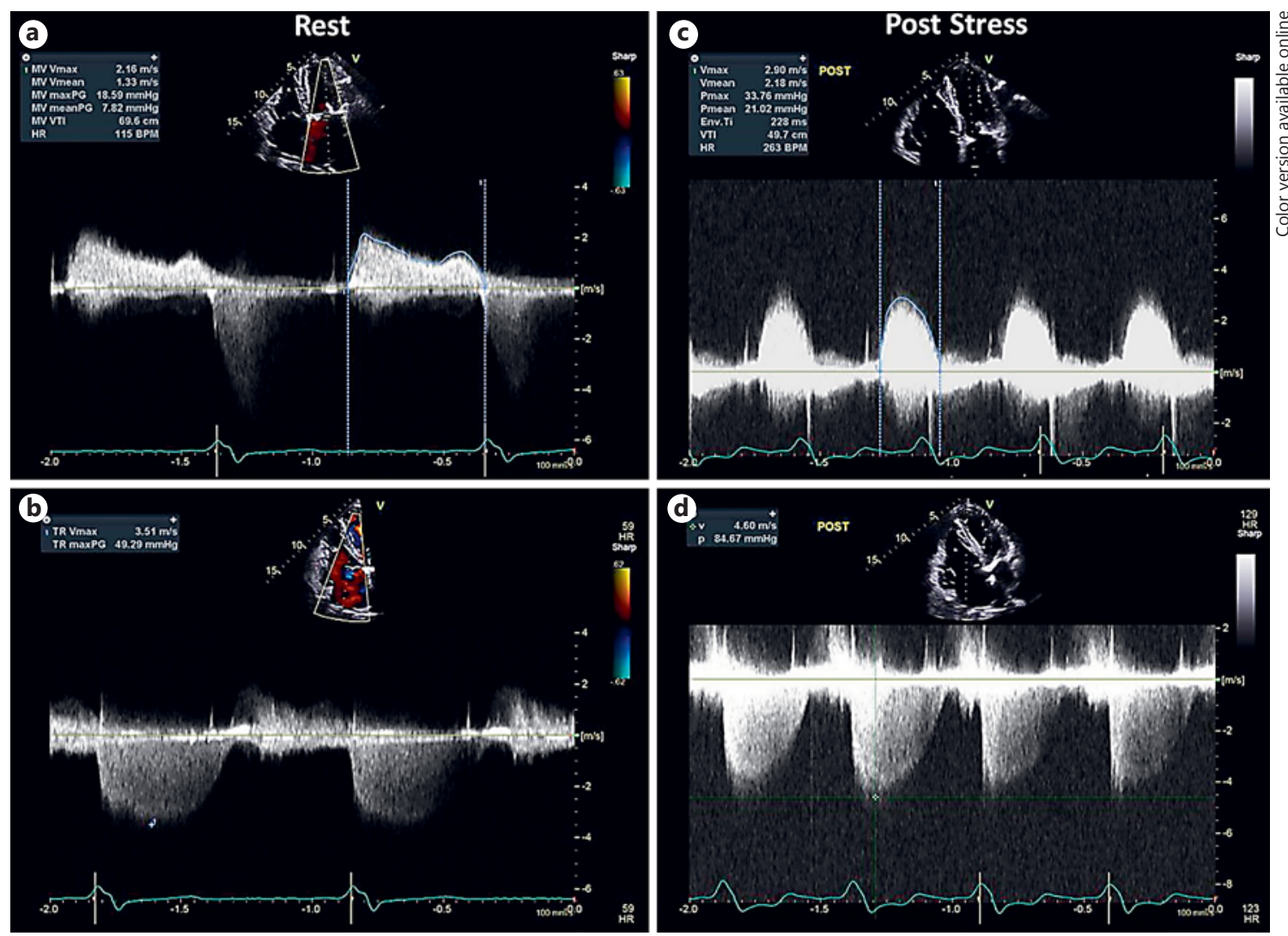

Fig. 4. Rest and exercise stress echocardiogram: severe mitral stenosis in a 68-year-old female with a history of rheumatic heart disease. a, b Rest peak and mean mitral valve (MV) gradients of 18.6 and $7.8 \mathrm{~mm} \mathrm{Hg}$ with a right ventricular systolic pressure (RVSP) of $49 \mathrm{~mm} \mathrm{Hg}$ at a heart rate (HR) of 59 beats/min. c, d Exercise stress with an increase in peak and mean mitral valve gradients to 33.7 and $21.0 \mathrm{~mm} \mathrm{Hg}$ with an RVSP of $84.5 \mathrm{~mm} \mathrm{Hg}$ at an approximate HR of 125 beats/min.

and reasonable (class IIa) if only moderate in severity regardless of LVEF. If the LVEF is $<30 \%$, MV surgery is reasonable (class IIa) if there is an option for revascularization of viable myocardium [7]. Lastly, the ESC and ACC/AHA agree that MV surgery can be considered (class IIb) for patients with severe secondary MR if their symptoms persist despite optimal medical therapy and alternative therapies where indicated, such as chronic resynchronization therapy, have failed to improve symptoms $[6,7]$.

\section{Role of Stress Echocardiography in Mitral}

\section{Regurgitation}

Stress echocardiography, preferably ESE, plays a pivotal role in patients with chronic MV regurgitation, particularly in circumstances where a discrepancy exists between the patients' symptoms and the severity of valvular regurgitation (i.e. asymptomatic severe MR or symptom- atic moderate MR). Observational studies in asymptomatic or minimally symptomatic (NYHA I or II) patients with significant MR (>moderate) demonstrate that 20 $30 \%$ of patients are found to have a reduced functional capacity, are associated with a higher risk of adverse events and worse longer-term outcomes, including allcause mortality (up to 12 years), without early surgery [43-45]. Based on exercise parameters alone, individuals who achieved $85-100$ and $<85 \%$ of age-/sex-predicted metabolic equivalents are associated with 2-3 times higher risks of all-cause mortality, myocardial infarction, stroke, and progression to congestive heart failure, respectively. Additionally, a lower heart rate recovery is also indicative of a worse outcome [46].

It is well documented that MR is a dynamic process. The severity and duration of MR may increase with exercise and may help explain the symptomatic deterioration and association with a higher risk of mortality and pro- 
Table 4. Mitral regurgitation (MR): abnormal stress echocardiography responses and contraindications

\begin{tabular}{|c|c|c|}
\hline & High-risk findings with stress & Contraindications \\
\hline Severe MR & $\begin{array}{l}\text { Asymptomatic, primary } \\
\text { Development of symptoms }{ }^{\mathrm{a}} \\
\text { Exercise capacity }<85 \% \text { of predicted for age/gender }{ }^{\mathrm{a}} \\
\text { Increase in RVSP }>60 \mathrm{~mm} \mathrm{Hg} \text { with exercise } \mathrm{b}^{\mathrm{b}} \\
\text { Latent left ventricular dysfunction } \\
\text { Onset of atrial fibrillation } \\
\text { Abnormal heart rate recovery } \\
\text { Asymptomatic, secondary } \\
\text { RWMA consistent with ischemic territory } \\
\text { Development of acute pulmonary edema without obvious cause } \\
\text { Effective regurgitant orifice area increase }>13 \mathrm{~mm}\end{array}$ & Severe symptomatic MR \\
\hline Moderate MR & $\begin{array}{l}\text { Symptomatic } \\
\text { Increase to severe MR }\end{array}$ & \\
\hline
\end{tabular}

RVSP, right ventricular systolic pressure; RWMA, regional wall motion abnormality.

${ }^{a}$ Indication for mitral valve surgery. ${ }^{b}$ European Society of Cardiology (ESC) indication for mitral valve surgery if high likelihood of durable repair and low surgical risk.

gression to heart failure in patients with at least $3+\mathrm{MR}$ [47]. Where this possibility is suspected, it is vital to assess valve severity using color Doppler techniques at peak exercise. This may occur in MR due to MV prolapse (especially bileaflet involvement), rheumatic disease, and especially when there is a component of LV outflow tract obstruction with systolic anterior motion of the valve. Rest studies can also fail to recognize high-risk features, including exercise-induced PHTN, latent LV dysfunction, onset of atrial fibrillation, and/or abnormal heart rate recovery.

Both ACC/AHA and ESC guidelines recommend use of stress echocardiography (IIa indication) to quantify exercise-induced changes in MR when a discrepancy exists among valve severity and the presence/absence of symptoms $[6,7]$. The ESC additionally recognizes the use of exercise echocardiography to quantify exercise-induced changes in MR, RVSP, and LV function and specifically recognizes the presence of exercise-induced PHTN (RVSP $>60 \mathrm{~mm} \mathrm{Hg}$ ) as a class IIb indication for surgery for asymptomatic patients with severe MR [7]. For those individuals who do not meet criteria for surgical intervention, the ACC/AHA guidelines also support use of ESE to establish an exercise tolerance baseline for comparison with future assessments (class IIa) [6].

$\mathrm{RV}$ function has also been increasingly recognized as an important prognostic tool. Specifically, exercise TAPSE, if reduced (<at least $19 \mathrm{~mm}$ ), was associated with earlier MV surgery in patients with significant $(>3+)$ asymptomatic MR [48]. Alterations in deformation with exercise can be useful to detect subclinical LV dysfunction. GLS at peak exercise, specifically GLS greater than $-18.5 \%$ and an exercise-induced change in GLS of $<1.9$, is predictive of postoperative LV dysfunction (LVEF $<50 \%$ ) [49]. Exercise-induced improvement in GLS $<2 \%$ was similarly found to be associated with a 2 -fold higher risk of cardiac events (cardiovascular death, MV surgery, and hospitalization for acute pulmonary edema or heart failure) [50]. Although not all of these high-risk features (Table 4) are indications for early surgical intervention, they may at least indicate that these individuals would benefit from a closer follow-up than would normally be indicated. Conversely, recent data for severe degenerative MR suggest that the presence of a preserved functional capacity as compared to those of similar age and gender may allow corrective surgery to be delayed by at least 1 year without risk of adversely affecting outcomes [45].

Stress echocardiography is also useful in patients with functional MR, especially in ischemic MR. In asymptomatic patients, ESE can identify patients with unrecognized symptoms but also reveal subclinical LV dysfunction secondary to silent ischemia. In symptomatic patients, ESE can objectively quantify symptoms, help establish an ischemic versus nonischemic etiology, and may assess the extent of myocardial viability to guide medical decision-making. Additionally, stress echocar- 
diography provides prognostic value for systolic heart failure patients with ischemic MR. Specifically an increase in the effective regurgitant orifice area of $\geq 13 \mathrm{~mm}^{2}$ with exercise is associated with increased morbidity, mortality, hospitalization for worsening heart failure, and major cardiac events. Similarly, patients with a larger increase in RVSP with exercise (29 vs. $17 \mathrm{~mm} \mathrm{Hg}$ ) are at higher risk of hospitalization for heart failure and cardiac death $[51,52]$.

\section{Prosthetic Valves}

Patients with prosthetic heart valves may develop exertional symptoms secondary to valve stenosis (leaflet calcification or pannus/thrombus formation) or regurgitation, patient-prosthesis mismatch (PPM), significant coronary artery disease, PHTN, and/or another primary pulmonary process. Normal and abnormal functioning prostheses can generate similar TPGs at rest. Therefore, stress echocardiography is important to confirm the presence or absence of hemodynamically significant prosthetic valve stenosis or PPM, especially when discordance exists among the patient's symptoms and prosthetic valve hemodynamics measured at rest. Additionally, prosthetic heart valves are inherently stenotic but can have normal TPGs at rest. Thus, valve dysfunction may only be recognized when evaluated with exertion [53].

During ESE, a disproportionate increase in mean TPGs compared to rest TPGs ( $>20 \mathrm{~mm} \mathrm{Hg}$ for aortic; $>12$ $\mathrm{mm} \mathrm{Hg}$ for mitral prosthesis) generally indicates a fixed obstruction either secondary to severe prosthesis dysfunction or PPM, especially if there is exact reproduction of the patient's reported symptoms. The 2009 American Society of Echocardiography Prosthetic Valve Guidelines recommend that evaluating for a significant obstruction should be similar to guidelines used for native stenotic valves as previously described, including evaluation for the presence and/or severity of PHTN that, if present, can further support the case for valve dysfunction or PPM. Additionally, nonprosthetic causes, such as coronary artery ischemia with exertion, can be identified [53].

An extremely important cause of exertional symptoms to recognize is PPM as it is essential during planning for subsequent valve replacements. PPM occurs when the effective orifice area (EOA) of the inserted prosthetic valve is too small relative to body size. For transvalvular gradients to remain low, the EOA must be proportionate in size to the patients' cardiac output. This is especially imperative to recognize in patients with a large body surface area and a small aortic annulus as their native annulus

Echocardiograpy in Valvular Heart Disease may not fit the size of prosthesis required to avoid PPM [54]. For this reason, PPM is more likely to be seen in patients with prostheses $<21 \mathrm{~mm}(\mathrm{AV})$ or $<25 \mathrm{~mm}$ (MV) than for larger prostheses [55]. When PPM is present, abnormally high TPGs become even more elevated during exercise secondary to an augmented cardiac output with a fixed EOA as described by the hydraulic equation in which the TPG is a square function of flow (Q) $\left(\mathrm{TPG}=\mathrm{Q}^{2} /\left[\mathrm{k} \times \mathrm{EOA}^{2}\right]\right)[56]$. The severity of PPM can be further quantified by indexing the EOA to body surface area (iEOA) with moderate PPM defined as an iEOA between 0.65 and $0.85 \mathrm{~cm}^{2} / \mathrm{m}^{2}$ and severe if $<0.65 \mathrm{~cm}^{2} / \mathrm{m}^{2}$ for the aortic position, and $<1.2$ and $<0.9 \mathrm{~cm}^{2} / \mathrm{m}^{2}$, respectively, for the mitral position $[54,55]$.

\section{Competitive Sports Eligibility}

Exercise tolerance testing can also be used to determine eligibility for participation in competitive sports with specifics outlined in Table 5 adapted from the ACC/ AHA 2015 Eligibility and Disqualification Recommendations for Competitive Athletes with Cardiovascular Abnormalities: Task Force 5: Valvular Heart Disease [57]. Additionally, exercise testing can elucidate cardiac adaptations and pathologic changes occurring in response to exercise in athletes experiencing symptoms during training or competition. Sports are classified by their increasing intensity of dynamic (I $\rightarrow$ III) or static exercise $(\mathrm{A} \rightarrow$ C) required to participate in that sport during competition (i.e., rowing represents a high-intensity dynamic and static exercise [IIIC] and bowling represents a low-intensity dynamic and static exercise [IA]). Dynamic exercise requires a significant increase in cardiac output achieved through an increase in heart rate and stroke volume (via an increase in end-diastolic volume and a decrease in end-systolic volume) resulting in a volume challenge to the heart. Conversely, static sports, as a result of contraction of large muscle groups, produce large fluctuations in afterload. There are no prospective clinical trials evaluating competitive athletes with valvular heart disease; therefore, recommendations on eligibility to participate is largely based on expert opinion. Current guidelines place significant emphasis on the use of exercise testing to a level commensurate with the degree of proposed activity to decide about eligibility to participate. We recommend using stress echocardiography as the optimal means of stress testing due to the additional information provided by the imaging component of the examination, including the ventricular response to exercise, change in severity of the valvular lesion with stress, and change in RVSP (Table 5). 
Table 5. 2015 ACC/AHA sports eligibility recommendations for asymptomatic athletes and the role of exercise testing

\begin{tabular}{|c|c|c|}
\hline Valve disease & Eligibility for competitive sports & $\begin{array}{l}\text { Exercise tolerance } \\
\text { testing }^{\text {a }}\end{array}$ \\
\hline \multicolumn{3}{|l|}{ Aortic valve } \\
\hline \multicolumn{3}{|l|}{ Aortic stenosis } \\
\hline Severe & None except possibly low-intensity sports: class IA sports (class III) & No \\
\hline Moderate & Low and moderate static/dynamic sports: classes IA, IB, IIA sports (class IIa) & Yes (class IIa) \\
\hline Mild & All sports (class IIa) & Yes (class IIa) \\
\hline \multicolumn{3}{|l|}{ Aortic regurgitation } \\
\hline Severe ${ }^{\mathrm{b}}$ & All sports (class IIb) & Yes (class IIb) \\
\hline LVEF $<50 \%$ or severe LV dilation ${ }^{c}$ & None (class III) & No \\
\hline Mild to moderate & All sports (class I) & Yes (class I) \\
\hline Moderate LV dilation $^{\mathrm{d}}$ & All sports (class IIa) & Yes (class IIa) \\
\hline \multicolumn{3}{|l|}{ Mitral valve } \\
\hline \multicolumn{3}{|l|}{ Mitral stenosis } \\
\hline Severe & None except possibly low-intensity sports: class IA sports (class III) & Yes (class I) \\
\hline Moderate & No specific recommendation given & Yes (class I) \\
\hline Mild & All sports (class IIa) & Yes (class I) \\
\hline \multicolumn{3}{|l|}{ Mitral regurgitation ${ }^{\mathrm{e}}$} \\
\hline Severe & No specific recommendation given; recommend same as below & Yes (class I) \\
\hline Mild LV enlargement ${ }^{\mathrm{f}}$ & Low- and some moderate-intensity sports: class IA, IIA, IB (class IIb) & Yes (class I) \\
\hline Moderate with mild LV enlargement ${ }^{\mathrm{f}}$ & All sports (class IIa) & Yes (class I) \\
\hline Mild to moderateg & All sports (class I) & Yes (class I) \\
\hline LV enlargement ${ }^{\mathrm{h}}$, PHTN, or LVEF $<60 \%$ & None except possibly low-intensity class IA sports (class III) & No \\
\hline Anticoagulation & None with risk of bodily contact (class III) & No \\
\hline \multicolumn{3}{|c|}{ 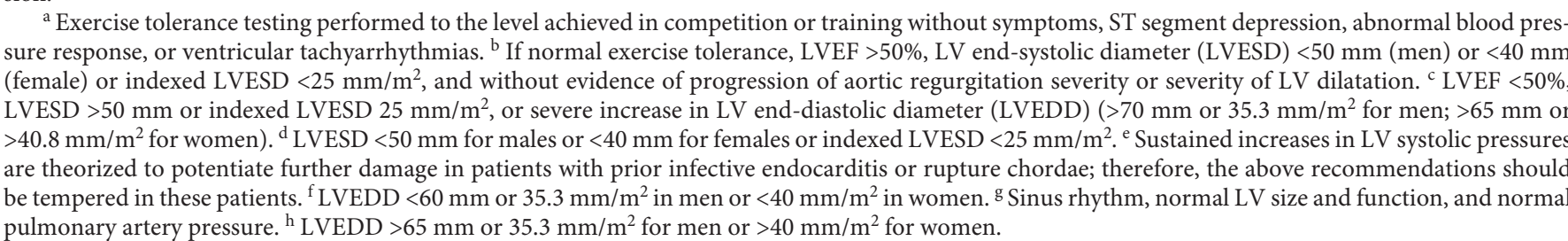 } \\
\hline
\end{tabular}

\section{Conclusions}

Stress echocardiography, both exercise and pharmacological, plays a vital and expanding role in the management of patients with valvular heart diseases. Its primary use remains in native and prosthetic left-sided valvular lesions to identify optimal timing for surgical or percutaneous valve intervention. Stress echocardiography not only identifies high-risk features that indicate the need for earlier surgery but also provides prognostic value for the peri- and postoperative period, appropriate diagnosis in the case of LFLG severe AS, and when a discrepancy exists between symptoms and valve severity, risk stratification to guide monitoring frequency, and guidance for eligibility in competitive sports participation. Although stress echocardiography is a valuable re- source in the evaluation of valvular heart disease, it is always important to keep in mind the limitations of the test, including technical difficulties and variations in measured values related to different loading conditions, sonographers, and/or ultrasound machines. As ACC/ AHA and ESC guidelines continue to recognize the value of stress echocardiography, future research should continue to focus on recognition of exercise-induced maladaptations and their impact on long-term outcomes.

\section{Conflict of Interest}

The authors have no disclosures, conflicts, or competing interests. 


\section{References}

1 Dhoble A, Sarano ME, Kopecky SL, et al: Safety of symptom-limited cardiopulmonary exercise testing in patients with aortic stenosis. Am J Med 2012;125:704-708.

2 Lancellotti P, Magne J: Stress echocardiography in regurgitant valve disease. Circ Cardiovasc Imaging 2013;6:840-849.

3 Lin SS, Roger VL, Pascoe R, et al: Dobutamine stress Doppler hemodynamics in patients with aortic stenosis: feasibility, safety, and surgical correlations. Am Heart J 1998;136:1010-1016.

4 van Le D, Jensen GV, Carstensen S, et al: Cardiopulmonary exercise testing in patients with asymptomatic or equivocal symptomatic aortic stenosis: feasibility, reproducibility, safety and information obtained on exercise physiology. Cardiology 2016;133:147-156.

5 Pierard LA, Lancellotti P: Stress testing in valve disease. Heart 2007;93:766-772.

6 Nishimura RA, Otto CM, Bonow RO, et al: 2014 AHA/ACC guideline for the management of patients with valvular heart disease: a report of the American College of Cardiology/American Heart Association Task Force on Practice Guidelines. J Thorac Cardiovasc Surg 2014;148:e1-e132.

7 Vahanian A, Alfieri O, Andreotti F, et al: Guidelines on the management of valvular heart disease (version 2012). The Joint Task Force on the Management of Valvular Heart Disease of the European Society of Cardiology (ESC) and the European Association for Cardio-Thoracic Surgery (EACTS) (in Italian). G Ital Cardiol (Rome) 2013;14:167-214.

8 Lancellotti P, Pellikka PA, Budts W, et al: The clinical use of stress echocardiography in non-ischaemic heart disease: recommendations from the European Association of Cardiovascular Imaging and the American Society of Echocardiography. Eur Heart J Cardiovasc Imaging 2016;17:1191-1229.

9 Yavagal ST, Deshpande N, Admane P: Stress echo for evaluation of valvular heart disease. Indian Heart J 2014;66:131-138.

10 Bhattacharyya S, Khattar R, Chahal N, et al: Dynamic assessment of stenotic valvular heart disease by stress echocardiography. Circ Cardiovasc Imaging 2013;6:583-589.

11 Otto CM, Burwash IG, Legget ME, et al: Prospective study of asymptomatic valvular aortic stenosis. Clinical, echocardiographic, and exercise predictors of outcome. Circulation 1997;95:2262-2270.

12 Kodali SK, Williams MR, Smith CR, et al: Two-year outcomes after transcatheter or surgical aortic-valve replacement. N Engl J Med 2012;366:1686-1695.

13 Makkar RR, Fontana GP, Jilaihawi $\mathrm{H}$, et al: Transcatheter aortic-valve replacement for inoperable severe aortic stenosis. N Engl J Med 2012;366:1696-1704.

14 Society of Thoracic Surgeons: Adult Cardiac Surgery Database: Executive Summary 10 Years STS Report. Durham, Duke Clinical Institute, 2010.
15 Masri A, Goodman AL, Barr T, et al: Predictors of long-term outcomes in asymptomatic patients with severe aortic stenosis and preserved left ventricular systolic function undergoing exercise echocardiography. Circ Cardiovasc Imaging 2016;9:e004689.

16 Rafique AM, Biner S, Ray I, et al: Meta-analysis of prognostic value of stress testing in patients with asymptomatic severe aortic stenosis. Am J Cardiol 2009;104:972-977.

17 Lancellotti P, Magne J, Donal E, et al: Determinants and prognostic significance of exercise pulmonary hypertension in asymptomatic severe aortic stenosis. Circulation Circulation.2012;126:851-859.

18 Marechaux S, Ennezat PV, LeJemtel TH, et al: Left ventricular response to exercise in aortic stenosis: an exercise echocardiographic study. Echocardiography 2007;24:955-959.

19 Donal E, Thebault C, O'Connor K, et al: Impact of aortic stenosis on longitudinal myocardial deformation during exercise. Eur J Echocardiogr 2011;12:235-241.

20 Clavel MA, Burwash IG, Mundigler G, et al: Validation of conventional and simplified methods to calculate projected valve area at normal flow rate in patients with low flow, low gradient aortic stenosis: the multicenter TOPAS (True or Pseudo Severe Aortic Stenosis) study. J Am Soc Echocardiogr 2010;23: 380-386.

21 Clavel MA, Ennezat PV, Marechaux S, et al: Stress echocardiography to assess stenosis severity and predict outcome in patients with paradoxical low-flow, low-gradient aortic stenosis and preserved LVEF. JACC Cardiovasc Imaging 2013;6:175-183.

22 Monin JL, Quere JP, Monchi M, et al: Lowgradient aortic stenosis: operative risk stratification and predictors for long-term outcome: a multicenter study using dobutamine stress hemodynamics. Circulation 2003;108: 319-324.

23 Lancellotti P, Karsera D, Tumminello G, et al: Determinants of an abnormal response to exercise in patients with asymptomatic valvular aortic stenosis. Eur J Echocardiogr 2008;9: 338-343.

24 Dumesnil JG, Pibarot P, Carabello B: Paradoxical low flow and/or low gradient severe aortic stenosis despite preserved left ventricular ejection fraction: implications for diagnosis and treatment. Eur Heart J 2010;31:281289.

25 Hachicha Z, Dumesnil JG, Bogaty P, et al: Paradoxical low-flow, low-gradient severe aortic stenosis despite preserved ejection fraction is associated with higher afterload and reduced survival. Circulation 2007;115: 2856-2864.

26 Bax JJ, Bonow RO, Tschope D, et al: The potential of myocardial perfusion scintigraphy for risk stratification of asymptomatic patients with type 2 diabetes. J Am Coll Cardiol 2006;48:754-760.
27 Leon MB, Smith CR, Mack M, et al: Transcatheter aortic-valve implantation for aortic stenosis in patients who cannot undergo surgery. N Engl J Med 2010;363:1597-1607.

28 Adams DH, Popma JJ, Reardon MJ, et al: Transcatheter aortic-valve replacement with a self-expanding prosthesis. N Engl J Med 2014;370:1790-1798.

29 Clavel M-A, Ennezat PV, Maréchaux S, et al: Stress echocardiography to assess stenosis severity and predict outcome in patients with paradoxical low-flow, low-gradient aortic stenosis and preserved LVEF. JACC Cardiovasc Imaging 2013;6:175-183.

30 Marechaux S, Hachicha Z, Bellouin A, et al: Usefulness of exercise-stress echocardiography for risk stratification of true asymptomatic patients with aortic valve stenosis. Eur Heart J 2010;31:1390-1397.

31 Borer JS, Hochreiter C, Herrold EM, et al: Prediction of indications for valve replacement among asymptomatic or minimally symptomatic patients with chronic aortic regurgitation and normal left ventricular performance. Circulation 1998;97:525-534.

32 Forsberg LM, Nylander E, Tamas E: Exercise echocardiography predicts postoperative left ventricular remodeling in aortic regurgitation. Scand Cardiovasc J 2014;48:4-12.

33 Wahi S, Haluska B, Pasquet A, et al: Exercise echocardiography predicts development of left ventricular dysfunction in medically and surgically treated patients with asymptomatic severe aortic regurgitation. Heart 2000;84: 606-614.

34 Bonow RO, Carabello BA, Chatterjee K, et al: ACC/AHA 2006 guidelines for the management of patients with valvular heart disease: a report of the American College of Cardiology/American Heart Association Task Force on Practice Guidelines (writing committee to revise the 1998 guidelines for the management of patients with valvular heart disease) developed in collaboration with the Society of Cardiovascular Anesthesiologists endorsed by the Society for Cardiovascular Angiography and Interventions and the Society of Thoracic Surgeons. J Am Coll Cardiol 2006;48: e1-e148.

35 Kusunose K, Agarwal S, Marwick TH, et al: Decision making in asymptomatic aortic regurgitation in the era of guidelines: incremental values of resting and exercise cardiac dysfunction. Circ Cardiovasc Imaging 2014;7: 352-362.

36 Ewe SH, Haeck ML, Ng AC, et al: Detection of subtle left ventricular systolic dysfunction in patients with significant aortic regurgitation and preserved left ventricular ejection fraction: speckle tracking echocardiographic analysis. Eur Heart J Cardiovasc Imaging 2015;16:992-999.
Echocardiograpy in Valvular Heart Disease
Cardiology 2017;137:137-150

DOI: $10.1159 / 000460274$ 
37 Helin LM, Tamas E, Nylander E: Preoperative longitudinal left ventricular function by tissue Doppler echocardiography at rest and during exercise is valuable in timing of aortic valve surgery in male aortic regurgitation patients. J Am Soc Echocardiogr 2010;23:387-395.

38 Henri C, Pierard LA, Lancellotti P, et al: Exercise testing and stress imaging in valvular heart disease. Can J Cardiol 2014;30:10121026.

39 Cheitlin MD: Stress echocardiography in mitral stenosis: when is it useful? J Am Coll Cardiol 2004;43:402-404.

40 Brochet E, Detaint D, Fondard O, et al: Early hemodynamic changes versus peak values: what is more useful to predict occurrence of dyspnea during stress echocardiography in patients with asymptomatic mitral stenosis? J Am Soc Echocardiogr 2011;24:392-398.

41 Reis G, Motta MS, Barbosa MM, et al: Dobutamine stress echocardiography for noninvasive assessment and risk stratification of patients with rheumatic mitral stenosis. J Am Coll Cardiol 2004;43:393-401.

42 Armstrong GP, Griffin BP: Exercise echocardiographic assessment in severe mitral regurgitation. Coron Artery Dis 2000;11:23-30.

43 Mentias A, Naji P, Gillinov AM, et al: Strain echocardiography and functional capacity in asymptomatic primary mitral regurgitation with preserved ejection fraction. J Am Coll Cardiol 2016;68:1974-1986.

44 Messika-Zeitoun D, Johnson BD, Nkomo V, et al: Cardiopulmonary exercise testing determination of functional capacity in mitral regurgitation: physiologic and outcome implications. J Am Coll Cardiol 2006;47:25212527.

45 Naji P, Griffin BP, Barr T, et al: Importance of exercise capacity in predicting outcomes and determining optimal timing of surgery in sig- nificant primary mitral regurgitation. J Am Heart Assoc 2014;3:e001010.

46 Naji P, Griffin BP, Asfahan F, et al: Predictors of long-term outcomes in patients with significant myxomatous mitral regurgitation undergoing exercise echocardiography. Circulation 2014;129:1310-13019.

47 Naji P, Asfahan F, Barr T, et al: Impact of duration of mitral regurgitation on outcomes in asymptomatic patients with myxomatous mitral valve undergoing exercise stress echocardiography. J Am Heart Assoc 2015;4:e001348.

48 Kusunose K, Popović ZB, Motoki H, Marwick TH: Prognostic significance of exercise-induced right ventricular dysfunction in asymptomatic degenerative mitral regurgitation. Circ Cardiovasc Imaging 2013;6:167176.

49 Lancellotti P, Cosyns B, Zacharakis D, et al: Importance of left ventricular longitudinal function and functional reserve in patients with degenerative mitral regurgitation: assessment by two-dimensional speckle tracking. J Am Soc Echocardiogr 2008;21:13311336.

50 Magne J, Mahjoub H, Dulgheru R, et al: Left ventricular contractile reserve in asymptomatic primary mitral regurgitation. Eur Heart J 2014;35:1608-1616.

51 Lancellotti P, Gerard PL, Pierard LA: Longterm outcome of patients with heart failure and dynamic functional mitral regurgitation. Eur Heart J 2005;26:1528-1532.

52 Lancellotti P, Troisfontaines P, Toussaint AC, et al: Prognostic importance of exercise-induced changes in mitral regurgitation in patients with chronic ischemic left ventricular dysfunction. Circulation 2003;108:17131717.

53 Zoghbi WA, Chambers JB, Dumesnil JG, et al: Recommendations for evaluation of prosthet- ic valves with echocardiography and Doppler ultrasound: a report from the American Society of Echocardiography's Guidelines and Standards Committee and the Task Force on Prosthetic Valves, developed in conjunction with the American College of Cardiology Cardiovascular Imaging Committee, Cardiac Imaging Committee of the American Heart Association, the European Association of Echocardiography, a registered branch of the European Society of Cardiology, the Japanese Society of Echocardiography and the Canadian Society of Echocardiography, endorsed by the American College of Cardiology Foundation, American Heart Association, European Association of Echocardiography, a registered branch of the European Society of Cardiology, the Japanese Society of Echocardiography, and Canadian Society of Echocardiography. J Am Soc Echocardiogr 2009;22: 975-1014; quiz 1082-1084.

54 Pisano C, D'Amico T, Palmeri C, et al: Valve prosthesis-patient mismatch: hemodynamic, echocardiographic and clinical consequences. Interact Cardiovasc Thorac Surg 2011;13: 606-610.

55 Picano E, Pibarot P, Lancellotti P, et al: The emerging role of exercise testing and stress echocardiography in valvular heart disease. J Am Coll Cardiol 2009;54:2251-2260.

56 Pibarot P, Dumesnil JG: Prosthesis-patient mismatch: definition, clinical impact, and prevention. Heart 2006;92:1022-1029.

57 Bonow RO, Nishimura RA, Thompson PD, et al: Eligibility and disqualification recommendations for competitive athletes with cardiovascular abnormalities: Task Force 5: Valvular Heart Disease: a scientific statement from the American Heart Association and American College of Cardiology. Circulation 2015; 132:e292-e297. 